\title{
Tattoos: what is their significance?
}

\section{Vivek Khosla, Verghese Joseph \& Harvey Gordon}

\begin{abstract}
SUMMARY
The prevalence of tattoos is higher among people with mental disorders and those likely to come in contact with mental health services. The motivations for acquiring a tattoo are varied and tattoos can give clues to the presence of particular psychiatric conditions and to the inner world of patients. Psychiatrists need to be aware of the health and safety issues surrounding the tattooing procedure and be able to give appropriate advice to their patients if they wish to acquire a tattoo. The issue of capacity must be considered. This may be particularly relevant for clinicians working with adolescents, the most common age group for acquiring tattoos. Owing to the high proportion of adults who subsequently regret their tattoos and the associated psychological and social distress, clinicians should be aware of methods of tattoo removal.
\end{abstract}

\section{DECLARATION OF INTEREST}

None.

A permanent tattoo is created by inserting a pigment just below the dermal-epidermal junction of the skin with a needle or a similar implement. The pigment is attacked by the immune system and sits in macrophages and fibroblast cells, producing permanent coloration of the skin. Historical records indicate that the practice of tattooing has been part of human culture since ancient times, with its popularity waxing and waning depending on the existing cultural norms.

The presence of a tattoo has often been considered to signify something about the bearer. As discussed below, previous literature shows some association with psychiatric disorders and criminality. In this article we explore the history of tattooing, motivations for acquiring a tattoo and its relevance to psychiatric practice. In occasional circumstances, psychiatrists may be required to advise a patient wanting a tattoo. It is worth noting that although tattooing is generally associated with body art and decorative body modification, it also has important cosmetic uses after surgery, for example in patients with breast cancer.

Tattoos vary enormously in design, location, number and content. There is no agreed classification of tattoos but one early attempt divides them into seven groups (Ferguson-Rayport 1955):
- identification tattoos (service emblems; personal information; key life events)

- love tattoos (idealised, sentimental or maternal love; pornographic images)

- bombastic and pseudo-heroic tattoos (Skull and crossbones, 'Death before Dishonour', powerful animals)

- inveighing fate (horse shoe with 'Good Luck'; 'Friday the 13th')

- religious and commemorative

- private symbols (of significance only to the individual)

- miscellaneous (animals, birds, flowers).

\section{Tattooing through history}

Body marking has been present continuously since the history of mankind, playing diverse roles at various times (Ferguson-Rayport 1955). Evidence from archaeological evacuations date some forms of body marking to as far back as $12000 \mathrm{BC}$. Among early nomadic tribes, the tattoo was used as a mark of identity or belonging to a group and the tattooed marks, such as tigers, monkeys and birds, were usually totemic in nature (Palermo 2004). Tattoos are reported to have been present in the ancient cultures of Egypt, China, Japan and India (Palermo 2004).

Knowledge of the early history of European tattooing is sketchy. Evidence from caves in Portugal and France suggests that tattooing was being performed as early as $6000 \mathrm{BC}$ (Levy 1979). The practice of tattooing declined to the point of disappearance in the Dark and Middle Ages in Europe, although it flourished among people who lived outside the law (Palermo 2004). Captain Cook introduced the word 'tattoo' from Polynesia to the Western world on his return from the South Pacific in the late 18th century (Post 1968; Raspa 1990; Palermo 2004). There has been a resurgence of tattooing in modern Western culture in the past three decades, with prevalence rates as high as $24 \%$ recorded among the general population (Laumann 2006).

\section{Motivations behind tattooing}

Compared with other body modifications such as body piercing, tattoos are unique because they are permanent. Motivations for acquiring a tattoo found in the literature are summarised in Box 1.
Vivek Khosla is a consultant forensic psychiatrist with the Oxfordshire and Buckinghamshire Mental Health Foundation NHS Trust. He has a wide range of interests in forensic psychiatry. Verghese Joseph is a consultant psychiatrist at Stamford Resource Centre,

Stamford. His research interests include understanding abnormalities in brain function in schizophrenia using the tools of functional magnetic resonance imaging and evoked response potentials. Harvey Gordon is a consultant forensic psychiatrist with the Oxfordshire and Buckinghamshire Mental Health Foundation NHS Trust and an honorary senior lecturer in forensic psychiatry in the Department of Psychiatry, University of Oxford. He previously worked at Broadmoor Hospital and Bethlehem Royal Hospital. He has a wide range of interests in general and forensic psychiatry. Correspondence Dr Vivek Khosla, Oxford Clinic, Oxfordshire and Buckinghamshire Mental Health Foundation NHS Trust, Littlemore Hospital, Oxford OX4 4XN.UK. Email:vivek.khosla@ obmh.nhs.uk 
BOX 1 Motivations for acquiring a tattoo

- Religious reasons

- Belonging to a group

- Exoskeletal defence

- To prove toughness

- Association with sexual immaturity, rebellion problems with identity and need to assert independence

- In females, to enhance the feminine image or intended as social resistance

- Fashion

Common motivations include fashion, to prove masculinity and toughness, or as means of asserting independence, but whatever the motivation, some form of non-verbal communication appears to be central to tattooing. The nature of the communication varies and is dependent on the inner world of the individual.

Human skin interacts with the surrounding environment. It has a protective function but it is also the conduit of positive and negative sensations to the nervous system. Palermo (2004) postulated that skin plays an important role in defining the 'self'. Its modification, for example by tattooing, is a form of non-verbal communication of inner ideas or conflict within oneself or with the outside world. Tattoos may therefore represent a person's attempt at self-expression and defining their individuality. Psychological aspects of a person such as sexual immaturity, rebellion and problems with identity (Taylor 1968) have been associated with acquiring tattoos. Low self-esteem has also been noted (Raspa 1990), leading some to conclude that tattoos are a defence mechanism against negative emotions.

Some authors have suggested that tattoos may be a form of 'exoskeletal' self-defence (Manuel 2002), signifying aggression and strength in the bearer. They are often seen in prison populations and include tattoos relating to the individual's aggressive violent past and gang affiliation, possibly acquired to ward off unwanted attention or assaults in prison.

At times people acquire tattoos in the belief that they beautify the body and make it more attractive. This is pertinent to an increase in the prevalence of tattoos as a fashion statement in recent decades. A study exploring motivations for women acquiring tattoos (Atkinson 2002) reported that they obtained tattoos to enhance the feminine image or as a form of social resistance. In the former case, the tattoos were generally subtle and hidden, whereas they were generally bold and on exposed parts of the body in the latter.
Throughout history, tattoos have been acquired for religious, magical, social and communal reasons (Scheinfeld 2007). Even today, in some parts of the world strong religious and superstitious significance is found in certain body markings. Muslim women in Yugoslavia mark their forehead with a cross to ward off evil. People in seafaring circles have a tattoo of a rooster and pig on either foot to protect against death (Post 1968), and in some cultures, such as Cambodia and Burma, tattoos are acquired to protect the bearer in a fight or a war (Scheinfeld 2007).

No significant differences have been reported in motivations for getting tattooed between psychiatric and non-psychiatric populations (Gittleson 1969, 1973; Buhrich 1982), although psychiatric patients with tattoos are more likely to be emotionally immature and have psychosexual disturbances (De los Santos Sanchez-Barudo 1985, cited in Palermo 2004). Tattoos are obtained at a young age in both groups. In a minority of cases, psychotic experiences (for example, auditory hallucinations and passivity phenomena) have led individuals to seek a tattoos (Gittleson 1969). Acquisition of tattoos following a traumatic event such as rape or by patients with post-traumatic stress disorder has been observed (Atkinson 2002; Manuel 2002).

Whatever the motivation, it seems that in the majority of cases, tattoos are obtained on impulse at a young age (Varma 1999), and studies on adolescents show an association between tattoos and risk-taking behaviour and impulsivity (Brooks 2003).

\section{Tattoos and psychiatry}

Evidence of a relationship between tattoos and psychiatric disorders comes from studies of psychiatric pathology in tattooed individuals in non-psychiatric settings (Lander 1943; Measey 1972) and of tattooed psychiatric populations (Ferguson-Rayport 1955; Gittleson 1969; Raspa 1990; Inch 1993; Williams 1998). Both types of study report higher rates of alcoholism, drug misuse, impulsivity, risk-taking behaviour and personality disorders in both men and women (Lander 1943; Post 1968; Measey 1972; Buhrich 1982; Raspa 1990; Inch 1993). These individuals are also more likely to have a history of self-harm, possibly as a result of a disordered personality and increased impulsivity (Goldstein 1979; Buhrich 1982).

\section{Neuropsychiatric disability}

The incidence of neuropsychiatric disability is reportedly higher in tattooed individuals. Lander 
\& Kohn (1943) reported a 50\% higher rejection rate of tattooed individuals at an army induction centre in the USA, with $58 \%$ of rejections of tattooed individuals occurring on the grounds of neuropsychiatric disability, in contrast to 38\% of non-tattooed individuals. A study by Kramish (reported in Ferguson-Rayport 1955) between 1944 and 1946 at an induction centre that screened 300 men per day found that over $90 \%$ of individuals with the 'love tattoo' were rejected because of neuropsychiatric disability.

\section{Prevalence in psychiatric populations}

The prevalence of tattoos in psychiatric populations is higher than in general populations and ranges from 15 to 39\% (Lander 1943; Ferguson-Rayport 1955; Gittleson 1969, 1973; Williams 1998). One large uncontrolled survey (Ferguson-Rayport 1955) of first-time admissions to a psychiatric hospital found the overall prevalence rate of tattoos to be $16 \%$, a finding subsequently replicated by Gittleson (1969), which found the prevalence rate to be $15 \%$. A more recent study (Williams 1998) of psychiatric admissions found an overall prevalence rate of $16 \%$. Psychiatric in-patients with tattoos appear to have a more chaotic and deprived upbringing than nontattooed controls. This includes more time spent in children's homes, fewer years of education and younger age on leaving home (Buhrich 1982). They also have more current social instability as regards accommodation and employment.

Tattooed individuals in psychiatric settings have a high prevalence of personality disorders. In the Ferguson-Rayport et al (1955) study, 10\% of those with tattoos had schizophrenia and 57\% had a personality disorder. A later study by Gittleson and colleagues (1969) of male psychiatric admissions to an acute ward reported $48 \%$ prevalence of personality disorder among tattooed patients. Buhrich \& Morris (1982), in a study of in-patient admissions, reported a prevalence of more than $30 \%$ for both schizophrenia and personality disorders among those with tattoos. In a study of remand prisoners, Birmingham et al (1999) reported an association between visible tattoos and lifetime prevalence of schizophrenia.

The prevalence of tattoos in female psychiatric in-patients is not as high as that in men, but, if present, can indicate psychiatric pathology. A study by Williams (1998) reported that only 5\% of women admitted to a psychiatric hospital had a tattoo. Gittleson (1969) found only two female patients with tattoos among 938 female admissions to a psychiatric hospital, both exhibiting disturbed personalities. A study of a community sample of women (Romans 1998) found that being tattooed was associated with more psychiatric symptoms, borderline personality characteristics as well as increased alcohol use. Childhood sexual abuse was reported more often in women with tattoos. A similar finding was reported in a case series by Inch \& Huws (1993).

\section{Psychiatric illness and location and number of tattoos}

The most common location of tattoos reported in the literature is the forearm. The available research into shape, size, location, arrangement and content of tattoos and their relationship to any particular psychiatric disorder is limited and inconclusive (Ferguson-Rayport 1955; Post 1968; Gittleson 1969; Measey 1972; Newman 1982; Birmingham 1999). Individuals with personality disorder are more likely to have multiple and visible tattoos when compared with patients with schizophrenia (Gittleson 1969), and there appears to be some association between the number of tattoos and presence of a personality disorder. Measey (1972) reported $82 \%$ prevalence of personality disorder in individuals with 16 or more tattoos. FergusonRayport et al (1955) studied characteristics of tattoos in a sample of psychiatric in-patients. They reported that individuals with personality disorder tended to have multiple tattoos, haphazardly arranged on the arms, legs or chest. These were carelessly carried out, of pornographic/bombastic nature and they expressed inner conflict and deviation from social norms. In comparison, individuals with schizophrenia had fewer tattoos, in inconspicuous locations. They were of a personally symbolic nature and expressed the individual's separation from the normal world. In both groups, tattoos had been acquired at a young age and, in patients with schizophrenia, before the onset of mental illness.

\section{Drug misuse}

Tattooed individuals have higher rates of drug misuse and dependence (Buhrich 1982; Williams 1998). One small study (Borokhov 2006) found that it was not uncommon for individuals misusing drugs to acquire tattoos either generally or specifically related to the drug misuse; for example, a tattoo in the cubital fossa indicating the spot where the injection is commonly made, to hide scars of sclerosed veins, or a tattoo of a syringe, poppy or marihuana plant.

\section{Adolescents}

The prevalence of tattoos in adolescents has been increasing, with population estimates indicating that up to $16 \%$ of adolescents have a 
tattoo (Armstrong 1994; Preti 2006). Studies of adolescents show an association between tattoos and risk-taking behaviour, impulsivity, self-harm and drug misuse (Brooks 2003). It has also been suggested that the presence of a tattoo may be a risk factor for suicide or accidental death (Dhossche 2000). Disordered eating (of bulimic type) has been found to be more prevalent in adolescent girls with tattoos and body piercings (Preti 2006), but this is not true of eating disorders per se. The relationship between tattooing and risk-taking behaviour in adolescents is thought to be complex and probably emanates from underlying psychological reasons, in some cases related to an individual's attempt to define their self-identity or to overcome previous trauma. Therefore, the presence of body modification such as tattooing should prompt the clinician to explore other risky behaviours.

\section{Tattoos and criminal behaviour}

Lombroso, an Italian psychiatrist and a criminal anthropologist of the late 19th century, considered tattoos to be a clear sign of intrinsic atavism, a tendency to conform to wild and primitive behaviour in the 'born delinquent' (Ferracuti 1996). Tattoos are common among both adult and adolescent prisoners (Post 1968; Newman 1982; Birmingham 1999; Strang 2000; Hellard 2007). Prevalence rates ranging from three to five times those in the general population have been reported (Strang 2000; Hellard 2007). The prevalence is even higher among adult and adolescent prisoners who have used intravenous drugs (Putnins 2002). Tattooed individuals, with or without a mental disorder, are more likely to have previous convictions than non-tattooed controls (Gittleson 1969, 1973; Buhrich 1982).

Are tattooed individuals more likely to offend or is tattooing part of a criminal subculture? The answer is probably yes to both the questions. There are abundant examples of criminal gangs using tattoos, often on exposed areas of the body (Mallon 1999), as a marker of affiliation and membership. It is therefore possible that tattoos reinforce criminal behaviour by allowing individuals to affiliate with criminal elements in gangs. On the other hand, it seems that individuals with certain psychological makeup who are more likely to engage in criminal behaviour are also more likely to acquire a tattoo, owing to shared risk factors. Prisoners with antisocial and sadistic personality types, as measured by the Millons Clinical Multiaxial Inventory, are more likely to obtain tattoos while in prison (Manuel 2002). In incarcerated adolescents, having a tattoo is associated with engaging in hostile and aggressive behaviour and risk of violent recidivism (Putnins 2002). Rates of alcoholism, drug misuse (Buhrich 1982; Raspa 1990; Williams 1998; Brooks 2003), impulsivity, risk-taking behaviour (Brooks 2003) and personality disorders (Ferguson-Rayport 1955; Yamamoto 1963; Post 1968; Gittleson 1969; Williams 1998) are high in both men and women bearing tattoos, and all these are risk factors for criminal behaviour.

There are other reasons why tattoos are so common among prisoners. They may represent a form of visual communication and a form of defence. They may indicate gang membership and criminal past, symbolise aggression and toughness or a form of exoskeletal defence (Manuel 2002). They may also be obtained to rebel against the authorities, deal with boredom or express one's creative side (Mallon 1999).

Tattooing among prisoners is a public health problem, with $20-30 \%$ of tattooed prisoners, both male and female, acquiring their tattoo in prison. Not surprisingly, the techniques and materials used are crude and such individuals are at high risk of acquiring blood-borne viruses such as hepatitis C (Strang 2000; Hellard 2007).

\section{Health, safety and legal issues relating to permanent tattooing}

\section{Medical complications}

If safely practised, tattooing does not carry a high risk of medical complications. However, problems can arise with unsafe practice (Box 2),

\section{BOX 2 Complications of tattooing}

- Localised scarring

- Severe localised swelling

- Allergic reactions (to inks, pigments and gloves)

- Localised infections

- Septicaemia

- Transmission of blood-borne diseases such as hepatitis $B$ and $C$, and HIV by unsterilised equipment

- Bleeding (patients on selective serotonin reuptake inhibitors may be susceptible to this due to their antiplatelet properties)

- Granulomas

- Keloid formation

- Complications during magnetic resonance imaging (transient burning sensation and swelling due to metallic pigments; interference on the scans)

(US Food and Drug Administration 2006; Health and Safety Executive/Local Authorities Enforcement Liaison Committee 2008) 
the most serious being transmission of bloodborne viruses such as hepatitis B and C, and HIV. Local regulation had to be implemented in the UK and The Netherlands following an outbreak of hepatitis B (Worp 2006) in the early 1980s. Despite such legislation, about one in ten individuals, particularly adolescents, prisoners and those with mental health problems, engage in high-risk behaviour such as tattooing themselves, getting tattooed by friends and using unsterile needles (Ferguson-Rayport 1955; Brooks 2003; Hellard 2007). This may be related to their inability to access a professional tattoo artist because of age or incarceration or to lack of awareness of the hazards of the procedure. Individuals with tattoos score high on impulsivity and risk-taking behaviour (Brooks 2003), and this may itself contribute to taking part in unsafe practices.

\section{Legislation}

Legislation relevant to tattooing in England and Wales (Health and Safety Executive/Local Authorities Enforcement Liaison Committee 2008) includes the Health and Safety at Work etc. Act 1974, the Local Government (Miscellaneous Provisions) Act 1982 (which has been recently amended by the Local Government Act 2003), the Tattooing of Minors Act 1969 (which prohibits tattooing of individuals under the age of 18 in Great Britain) and the Medicines Act 1968. A tattooist could potentially be charged with assault if the procedure is carried out on an individual unable or incapable of giving valid consent.

\section{Practice and hygiene}

Tattooists normally work in tattoo studios or parlours. Their premises must be registered with the local environmental health department in England and Wales under the Local Government (Miscellaneous Provisions) Act 1982. It is illegal for an artist not to be registered. There is no specific training to become a tattooist; instead, an apprenticeship with a registered tattooist lasting $2-3$ years is undertaken. Tattooists must have skills to undertake a pre-procedure consultation, give verbal and written information about the procedure, and record information, including a medical history. Medical factors to consider include chronic skin conditions (such as eczema and psoriasis), haemophilia, heart disease, high/ low blood pressure, medication, epilepsy, diabetes, allergies, pregnancy and breastfeeding.

Tattooists are expected to maintain a good standard of personal hygiene as well as cleanliness of the premises, and take precautions to minimise any risk of infection, blood borne or otherwise. It

is recommended that they are immunised against tetanus and hepatitis B. It is strongly recommended that single-use equipment is used wherever feasible. When reusable equipment is used, facilities for steam sterilisation must be available. Tattooists should have appropriate procedures for disposing of clinical waste and sharps. They are not permitted to use injectable anaesthetics. Ethyl chloride spray is sometimes used as topical anaesthesia but is not thought to have any significant anaesthetic effect (Worp 2006).

\section{Tattoo removal}

Tattoo removal may be requested for personal reasons such as regret after having acquired a tattoo, to improve self-esteem, appear more professional or increase credibility (Varma 1999). Removal is sometimes required because of medical complications such as allergic or granulomatoustype reactions (Burris 2007). Removal methods (Box 3) are expensive and can lead to incomplete removal and scarification.

Gittleson (1973) described tattooing as a 'group activity of youth and regretted in maturity'. Up to $50 \%$ of those who acquire tattoos subsequently regret it, with duration of regret varying from 2 months to 48 years before requesting removal (Varma 1999). This is not surprising considering that the majority of tattoos are obtained impulsively at a young age (Varma 1999). A majority of adolescents report feeling positive about their body modification(s) (Brooks 2003), but a substantial proportion regret the decision with age. Gittleson $\&$ Wallen (1973) reported that $24 \%$ of men (mean age 52.4 years) wanted their tattoo(s) removed and

\section{BOX 3 Tattoo removal techniques}

$\begin{array}{ll}\begin{array}{l}\text { Salabrasion } \\ \text { Mechanical method which involves abrading } \\ \text { superficial dermis with coarse granules of } \\ \text { common table salt and a moist gauze pad. }\end{array} & \begin{array}{l}\text { inserted in the skin following trauma/injury } \\ \text { such as a fall, a car accident or damage } \\ \text { from an exploding firework). }\end{array} \\ \begin{array}{l}\text { It is effective for removing amateur tattoos. } \\ \text { Associated with risk of scarring. }\end{array} & \begin{array}{l}\text { Ching compounds such as tannic acid or } \\ \text { silver nitrate, the skin is punctured and the } \\ \text { chemicals are applied to create a scar. It can }\end{array} \\ \begin{array}{ll}\text { Dermabrasion } & \text { be used for amateur tattoos, but it is rarely }\end{array} \\ \begin{array}{ll}\text { Another mechanical method involving } \\ \text { tissue destruction using a wire brush or }\end{array} & \begin{array}{l}\text { used nowadays. } \\ \text { rapidly spinning diamond fraise wheel. }\end{array} \\ \begin{array}{l}\text { It is sometimes used in conjunction with } \\ \text { surgical excision. The procedure carries risk } \\ \text { of scarring. }\end{array} & \begin{array}{l}\text { Laser removal (thermal method) } \\ \text { This has become the treatment of choice. }\end{array} \\ \begin{array}{l}\text { Surgical excision } \\ \text { Suitable for small decorative, cosmetic or }\end{array} & \begin{array}{l}\text { treatments may be required. It is a relatively } \\ \text { expensive treatment. Side-effects include } \\ \text { hypo- or sometimes hyperpigmentation. }\end{array} \\ \text { 'traumatic' tattoos (where pigment becomes } & \end{array}$

Salabrasion

Mechanical method which involves abrading superficial dermis with coarse granules of . It is effective for removing amateur tattoos. risk of scarring.

\section{Dermabrasion}

Another mechanical method involving tissue destruction using a wire brush or rapidy spinning diamond fraise wheel.

Suitable for small decorative, cosmetic or 'traumatic' tattoos (where pigment becomes 
MCO answers

$1 \mathrm{~b} \quad 2 \mathrm{~b} \quad 3 \mathrm{e} \quad 4 \mathrm{a} \quad 5 \mathrm{e}$
$69 \%$ regretted acquiring a tattoo. This applies to both psychiatric and non-psychiatric populations (Gittleson 1969).

Tattoo removal, despite the difficulties, can be beneficial. Negative attitudes to a permanent body modification, especially if on an exposed part of the body, can be intense and cause social and psychological difficulties (Varma 1999) that can be alleviated by removal. Many people have a negative perception of individuals bearing tattoos, particularly visible ones, and therefore removal can help the individual in obtaining employment and gaining the social acceptance of others. In some cases, removal might indicate a profound positive change in the person, for example removal of a gang-related tattoo indicating an end to such associations (Mallon 1999).

There are mechanical, chemical and thermal methods (Burris 2007) for removing tattoos (Box 3). In some cases, tattoos can be camouflaged by injecting skin-tone pigments or by acquiring a new pattern on an existing tattoo.

In England and Wales, primary care trusts have commissioning policies for aesthetic treatments under which tattoo removal falls. Tattoo removal only for cosmetic purposes is not available on the National Health Service (NHS) unless the tattoo is the result of trauma (Box 3). It is considered a low-priority treatment, but some trusts may offer treatment on the basis of significant psychological distress and dysfunction likely to improve with treatment, or if the individual was a minor lacking 'Gillick competence' (of insufficient understanding and intelligence to be capable of making a reasonable decision) at the time of tattooing. Such treatments are approved on a case-by-case basis.

\section{Implications for clinical practice}

The relevance of tattoos needs to be seen in the context of the prevailing culture. With increasing popularity of the practice and increasing prevalence, a large proportion of individuals with tattoos will be of little significance to a psychiatrist. However, tattoos are overrepresented in marginal groups in society, which often come in contact with the psychiatric profession, whether in the community, in-patient or prison setting, and can provide clinically useful information. Their presence and type can give clues to the existence of particular psychiatric conditions and to the inner world of patients. Tattoos are sometimes acquired for a variety of complex psychological reasons, exploration of which in a therapeutic setting can prove useful. If seen as a form of non-verbal communication, they give clues to important personality traits (such as impulsiveness and risk-taking) that can be relevant to general lifestyle and clinical treatment. We recommend that tattoos are noted during the psychiatric examination and a record is made of their characteristics (location, size and content). The reasons and motivation for acquiring the tattoo(s) should be explored. Another benefit of recording tattoos is that they can be used for person identification.

Psychiatrists need to be aware of the medical complications surrounding the procedure of tattooing and be able to give appropriate advice to their patients if they wish to acquire a tattoo. The issue of capacity must be considered in a patient seeking advice on obtaining a tattoo. For example, a patient's wish to obtain a tattoo may be related to their disturbed mental state. In such circumstances, there is a potential risk that a patient will subsequently bring legal action against the doctor, if they were allowed to obtain a tattoo while in an altered mental state. The assessment of capacity is particularly relevant to clinicians working with adolescents, the most common age group for acquiring tattoos. Owing to the high proportion of adults subsequently regretting their tattoos and the associated psychological and social distress, clinicians should be aware of the methods of tattoo removal.

\section{References}

Armstrong ML, McConnell C (1994) Tattooing in adolescents, more common than you think: the phenomenon and risks. Journal of School of Nursing 10: 22-9.

Atkinson M (2002) Pretty in ink: conformity, resistance, and negotiation in women's tattooing. Sex Roles 47: 219-35.

Birmingham L, Mason D, Grubin D (1999) The psychiatric implications of visible tattoos in an adult male prison population. Journal of Forensic Psychiatry 10: 687-95.

Borokhov A, Bastiaans R, Lerner V (2006) Tattoo designs among drug abusers. Israel Journal of Psychiatry Related Science 43: 28-33.

Brooks TL, Woods ER, Knight JR, et al (2003) Body modification and substance use in adolescents: is there a link? Journal of Adolescent Health 32: 44-9.

Buhrich N, Morris G (1982) Significance of tattoos in male psychiatric patients. Australian and New Zealand Journal of Psychiatry 16: 185-9.

Burris K, Kim K (2007) Tattoo removal. Clinics in Dermatology 25: 388-92.

Dhossche D, Snell KS Larder S (2000) A case-control study of tattoos in young suicide victims as a possible marker of risk. Journal of Affective Disorders 59: 165-8.

Ferguson-Rayport SM, Griffith RM, Straus EW (1955) The psychiatric significance of tattoos. Psychiatric Quarterly 29: 112-31.

Ferracuti S (1996) Cesare Lombroso (1835-1907). Journal of Forensic Psychiatry 7: 130-49.

Gittleson NL, Wallen GDP, Dawson-Butterworth K (1969) The tattooed psychiatric patient. British Journal of Psychiatry 115: 1249-53.

Gittleson NL, Wallen GDP (1973) The tattooed male patient. British Journal of Psychiatry 122: 295-300.

Goldstein N (1979) Psychological implications of tattoos. Journal of Dermatological Surgery and Oncology 5: 883-8. 
Health and Safety Executive/Local Authorities Enforcement Liaison Committee (2008) Local Authority Circular: Health and safety issues related to body piercing, tattooing and scarification (LAC 76/2). Health and Safety Executive/Local Authorities Enforcement Liaison Committee (http://www.hse.gov.uk/lau/lacs/76-2.htm).

Hellard ME, Aitken CK, Hocking JS (2007) Tattooing in prisons - not such a pretty picture. American Journal of Infection Control 35: 477-80.

Inch H, Huws R (1993) Tattoed female psychiatric patients. British Journal of Psychiatry 162: 128-9.

Lander J, Kohn HM (1943) A note on tattooing among selectees. American Journal of Psychiatry 100: 326-7.

Laumann AE, Derrick AJ (2006) Tattoos and body piercings in the United States: a national data set. Journal of the American Academy of Dermatology 55: 413-21.

Levy J, Sewell M, Goldstein N (1979) A short story of tattooing. Journal of Dermatological Surgery and Oncology 5: 851-6.

Mallon WK, Russell MA (1999) Clinical and forensic significance of tattoos. Top Emergency Medicine 21: 21-9.

Manuel L, Retzlaff PD (2002) Psychopathology and tattooing among prisoners. International Journal of Offender Therapy and Comparative Criminology 46: 522-31.

Measey LG (1972) The psychiatric and social relevance of tattoos in Royal Navy detainees. British Journal of Criminology 12: 182-6.

Newman G (1982) The implications of tattooing in prisoners. Journal of Clinical Psychiatry 42: 231-4.

Palermo GB (2004) Tattooing and tattooed criminals. Journal of Forensic Psychology Practice 4: 1-25.

Post RS (1968) The relationship of tattoos to personality disorders. Journal of Criminal Law, Criminology and Police Science 59 : $516-24$
Preti A, Pinna C, Nocco S, et al (2006) Body of evidence: tattoos, body piercing, and eating disorder symptoms among adolescents. Journal of Psychosomatic Research 61: 561-6.

Putnins A (2002) Young offenders, tattoos and recidivism. Psychiatry, Psychology and Law 9: 62-8.

Raspa RF, Cusack J (1990) Psychiatric implications of tattoos. American Family Physician 41: 1481-6.

Romans SE, Martin JL, Morris EM, et al (1998) Tattoos, childhood sexual abuse and adult psychiatric disorder in women. Archives of Women's Mental Health 1: 137-41

Scheinfeld N (2007) Tattoos and religion. Clinical Dermatology 25 362-6.

Strang J, Heuston J, Whiteley C, et al (2000) Is prison tattooing a risk behaviour for HIV and other viruses? Results from a national survey of prisoners in England and Wales. Criminal Behaviour and Mental Health 10: $60-6$.

Taylor AJW (1968) A search among borstal girls for the psychological and social significance of their tattoos. British Journal of Criminology 8: $170-85$.

US Food and Drug Administration (2006) Tattoos \& permanent makeup. US Department of Health and Human Services (http://www.cfsan.fda. gov/ dms/cos-204.html).

Varma S, Lanigan SW (1999) Reasons for requesting laser removal of unwanted tattoos. British Journal of Dermatology 140: 483-5.

Williams K (1998) Tattoos, scars, body adornment and dishevelment in an acute psychiatric population. Psychiatric Bulletin 22: 94-6.

Worp J, Boonstra A, Coutinho RA, et al (2006) Tattooing, permanent makeup and piercing in Amsterdam: guidelines, legislation and monitoring. Eurosurveillance 11: 34-6.

Yamamoto J, Seeman W, Lester BK (1963) The tattooed man. Journal of Nervous and Mental Disorder 136: 365-7.

\section{MCOs}

Select the single best option for each question stem

1 The term 'tattoo' was introduced to the Western world from Polynesia by:

a Captain Scott

b Captain Cook

c Pope Hadrian 1

d Nero

e Emperor Constantine.

2 Individuals with tattoo(s) do not have higher reported rates of:

a drug misuse

b obsessive-compulsive disorder

c personality disorder

\section{d impulsivity}

e alcohol misuse.

\section{Tattoo artists working legally in the UK} must be registered with the:

a local council

b local government

c health and safety executive

d tattoos association

e local environmental health department.
4 Which of the following is not a relative contraindication for tattooing:
a previous tattoos
b chronic skin conditions

\section{c haemophilia \\ d diabetes \\ e high/low blood pressure.}

5 Which of the following is true:

a tattoos are usually obtained in late adulthood

b only a minority of individuals regret acquiring a tattoo

c tattoos can be easily removed

$\mathrm{d}$ tattoo removal for cosmetic purposes is widely available on the NHS

e tattoo removal may be requested to improve self-esteem. 